\section{Erdheim-Chester Disease with Chest Wall Involvement}

Sir,

Erdheim-Chester Disease (ECD) is a rare systemic disease characterised by multifocal infiltration of histiocytes in bones with or without involvement of multiple organs. Clinical presentations of ECD vary from bone involvement to life-threatening multisystemic disease. Its pathogenesis is still unclear, but it is characterised by clonal expansion of histiocytes. Symptoms and signs of ECD depend on the location and extent of such involvement. The underlying cause of ECD is unclear. It is common between the ages of 40-60 years. Here, we present a patient who was diagnosed with $E C D$, as a result of chest wall involvement.

In the physical examination of a 41-year male patient with a complaint of chest pain admitted to our polyclinic, a mass on the chest wall was found. An expansion was detected in the right 4 th rib of the patient in chest computed tomography (CT) (Figure 1). The patient was operated and the lesion was excised $4 \mathrm{~cm}$ wide proximally and distally. Histopathological examination showed distinctive infiltration of ECD. The patient was discharged on the third postoperative day without any complication.

ECD described by Jakob Erdheim and William Chester in 1930, is a rare inflammatory disease with unknown etiology, characterised by organ infiltration of nonLangerhans foamy histiocytes (CD68 +, CD1a- negative sign) (Figure 2) ${ }^{1,2}$ Depending on the distribution of lesions, the clinical manifestations of EDC are varied. The disease primarily involves long bones (especially the distal femur, proximal tibia and fibula), but can involve all organs and tissues. According to the involved organ, it has a spectrum of presentations ranging from asymptomatic to life-threatening illness. Bone involvement is present in $96 \%$ of patients but symptomatic in $50 \%$. More than $50 \%$ of cases have non-skeletal internal organ involvement. ${ }^{3}$ Correspondingly, exophthalmos, papilledema, xanthelesma and papulonodular skin lesions, diabetes insipidus, severe pulmonary diseases, renal insufficiency, retroperitoneal involvement, cardiomyopathy, and disorders due to central nervous system involvement (pyramidal disorders, congnitive impairment, headache, etc.) are seen. ${ }^{3}$ Treatment should be multidisciplinary depending on clinical involvement.

Patients with chest tumor usually present with palpable mass. Asymptomatic patients are generally diagnosed on imaging studies during examinations, usually for unrelated reasons. Based on the growth of the tumor,

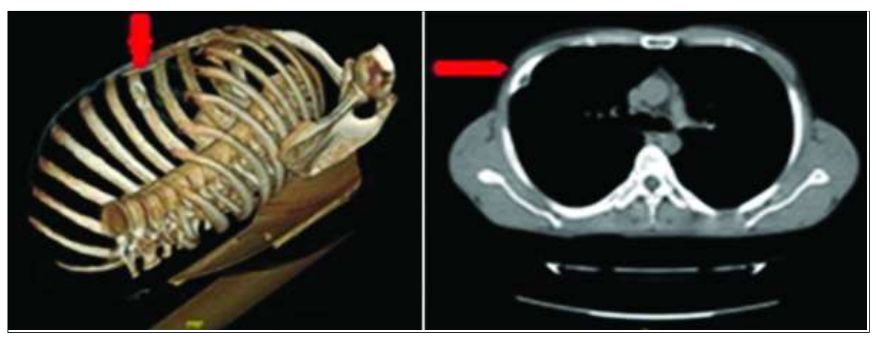

Figure 1: Preoperative 3D-CT and 2D-CT.

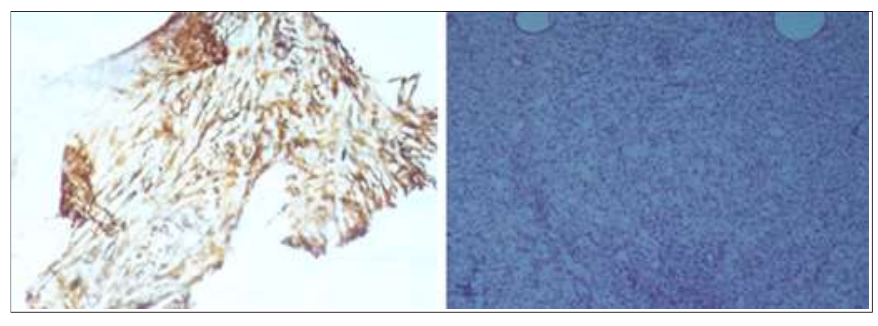

Figure 2: Typical histological finding; SMA infiltration and foamy histiocytes.

the symptoms develop due to invasion of adjacent structures. If invasion occurs in the neurological structures, paresthesiae and loss of strength occur. Symptoms such as fever, fatigue, weight loss may be seen. Chest X-ray, CT chest and magnetic resonance imaging (MRI) are used in the diagnosis. The exact diagnosis is made by histopathologic examination. There are no universally accepted guidelines for the diagnosis and treatment of ECD. Chemotherapy and/or radiotherapy is performed according to the pathological diagnosis after surgical resection. ECD is diagnosed upon a thorough clinical evaluation, a detailed patient history of symptoms, and high clinical surpicion in addition to specialised tests.

\section{REFERENCES}

1. Haroche J. Cardiovascular involvement, an overlooked feature of Erdheim Chester disease: report of 6 new cases and a literature review. Medicine 2004; 83:371-92.

2. Cavalli G. The multifaceted clinical presentations and manifestations of Erdheim-Chester disease: comprehensive review of the literature and of 10 new cases. Ann Rheum Dis 2016; 72:1691-5.

3. Adawi M, Bisharat B, Bowirrat A. Erdheim-Chester disease: Case report, clinical and basic investigations, and review of literature. Medicine 2016; 95:e5167.

Mehmet Akif Tezcan and Ibrahim Ethem Ozsoy

Department of Thoracic Surgery, Kayseri City Training and Research Hospital, Kayseri, Turkey

Correspondence: Dr. Mehmet Akif Tezcan, Department of

Thoracic Surgery, Kayseri City Training and Research

Hospital, Kayseri, Turkey

E-mail: mehmetakiftercan@gmail.com

Received: May 31, 2018; Accepted: September 13, 2018

$$
\text { ....饭... }
$$

